\title{
Multilayer Strip Dipole Antenna Using Stacking Technique and Its Application for Curved Surface
}

\author{
Charinsak Saetiaw and Chanchai Thongsopa \\ School of Telecommunication Engineering, Suranaree University of Technology, Thailand \\ Correspondence should be addressed to Charinsak Saetiaw; charinsak_s@yahoo.co.th and Chanchai Thongsopa; chan@sut.ac.th
}

Received 31 July 2013; Revised 14 November 2013; Accepted 20 November 2013

Academic Editor: Z. N. Chen

Copyright (c) 2013 C. Saetiaw and C. Thongsopa. This is an open access article distributed under the Creative Commons Attribution License, which permits unrestricted use, distribution, and reproduction in any medium, provided the original work is properly cited.

\begin{abstract}
This paper presents the design of multilayer strip dipole antenna by stacking a flexible copper-clad laminate utilized for curved surface on the cylindrical objects. The designed antenna will reduce the effects of curving based on relative lengths that are changed in each stacking flexible copper-clad laminate layer. Curving is different from each layer of the antenna, so the resonance frequency that resulted from an extended antenna provides better frequency response stability compared to modern antenna when it is curved or attached to cylindrical objects. The frequency of multilayer antenna is designed at $920 \mathrm{MHz}$ for UHF RFID applications.
\end{abstract}

\section{Introduction}

In the last decade, the technology of Radio Frequency Identification (RFID) has been used in many aspects of daily life including the logistic system, object tracking system, inventory management, human monitoring, and electronic banking [1]. RFID is a very valuable technology tool that holds the promise of replacing existing identification technologies like the bar code because it can provide longer reading distance, better reading speed, and large information storage capability. RFID system consist of reader and tag. A reader broadcasts a radio signal in its wireless transmission range for queried information contained in tag. After that, the tag will reply with required information. The detection range and accuracy are directly dependent on the performance of reader/tag antennas. However, RFID tag antenna has a lot of different types and shapes applicable with various kinds of products. The tag antenna can be printed onto flexible substrates (or the so-called inlays). The flexible substrates of the tag antenna have been affected by various distortions when applying to different packages on nonflat surface. This distortion in the structure will usually change the performance of the RFID tag antenna and the performance degradation in the RFID system, especially when operating in very high frequency.

A problem of antenna design is that it is difficult to cover all requirements, especially on different types of surface used if there are techniques to make an antenna tune itself by changing some properties depending on different environments. This technique will be efficient for a manufacturer and eventually for user as it is easily used in all types of application. A technique of stacking layer called multilayer antenna has been purposed. The proposed RFID tag antenna is operated in the ultra-high frequency (UHF) industrialscientific-medical (ISM) band around $900 \mathrm{MHz}$. UHF RFID is most popular because of its best appropriate properties such as size and reading range especially low cost and easy fabricate. A design of multilayer strip dipole antenna has been demonstrated of a purpose of this technique used in antenna design that make an antenna tune itself on different types of surface especially on curved surface.

In this paper, we purpose a novel UHF tag antenna with stacking technique suitable to be applied to curved surface or cylindrical object to resolve the performance degradation. We introduced a flexible material structure of copper clad laminate with dielectric substrate stacking on layer by layer that has better frequency response stability than single layer and suitable for RFID applications. However, the tag RFID is mostly used as strip dipole antenna [2,3]. Practically, strip dipole antenna property and efficiency including resonance frequency will be changed depending on the surface of the object on which it is laid, which may be rough or curved surfaces [4]. The result of curving will change the effective 


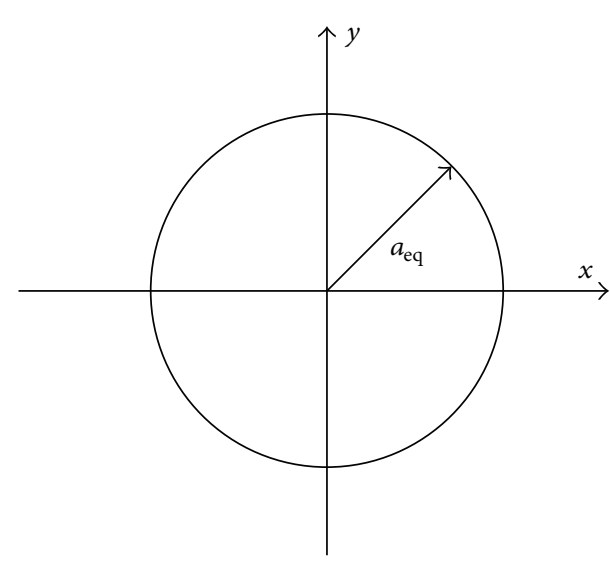

(a)

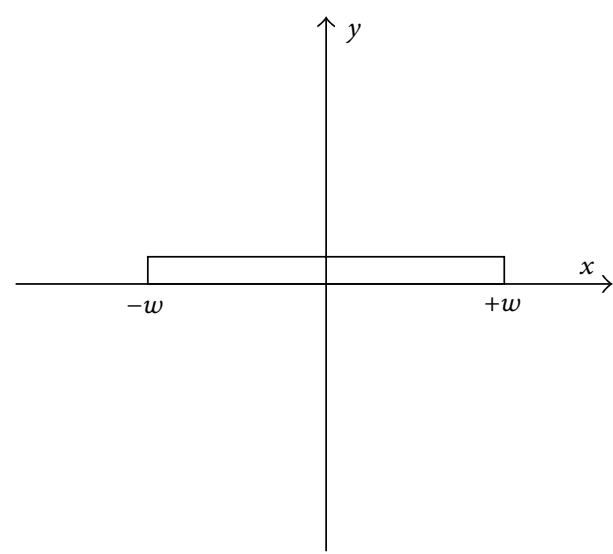

(b)

FIGURE 1: Schematic of cross section for thin-wire dipole antenna and microstrip dipole antenna.

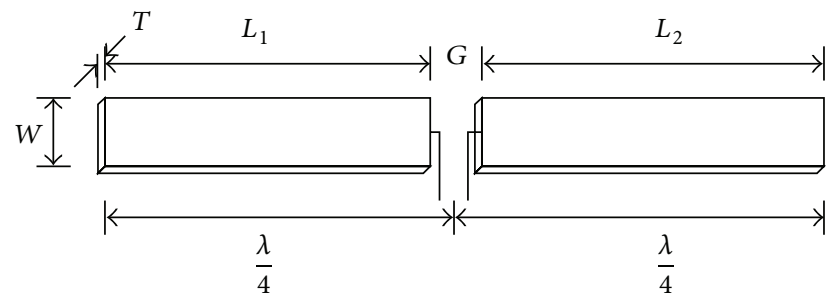

FIGURE 2: Schematic of strip dipole antenna.

length of antenna and affect the resonance frequency of antenna [5]. Multilayer strip dipole antenna will reduce the effect of curving based on relative changes in length on each layers caused by curving of the antenna which is not the same on each layer of antenna. So, the result of an extended length will prove a resonance frequency of antenna and better efficiency compared to modern antenna. The simulation and measurement of curving the RFID tag antenna models by using a multilayer strip dipole antenna will be discussed.

This paper is organized as follows. The first section is an introduction of RFID as already mentioned above. The second section is a demonstration of the antenna structural design and discussion on single-layer strip dipole antenna and multilayer strip dipole antenna. In simulation section, a reflection coefficient of curved antenna in various radiuses and different types of antenna are presented as a result of simulations. The next section presents a measurement result from prototype of the single-layer, triple-layers and five-layers antennas with straight and curved. The last section is the conclusion of the paper.

\section{Multilayer Strip Dipole Antenna}

In this section, a brief review of strip dipole antenna model and design is given. Then, the proposed multilayer strip dipole antenna with the effect of curving antenna is discussed.

Dipole antenna was designed in the late 19th century by Heinrich Hertz Rudolph [6]. The components of the dipole antenna connector consist of two straight lines of equal length lying on the same axis, separated by a small gap. The main factor for determining the frequency is the length of the antenna. The number of half wave length will be used for this purpose. After that, a design of thin-wire dipole antenna can be adapted to strip dipole antenna as shown in Figure 1.

From [7] we will have the relation of thin-wire dipole diameter $\left(a_{\mathrm{eq}}\right)$ compared to strip dipole antenna width $(w)$ as

$$
a_{\mathrm{eq}}=\frac{2 w}{4} .
$$

By this equation, we can design any strip dipole antenna that has some properties related to thin-wire dipole. Otherwise, the strip dipole antenna length will relate to their frequency designed.

Basically, rectangular strip antenna can be classified into two main categories depending on their length-to-width ratio. An antenna with a narrow rectangular strip (typically strip width less than $0.05 \lambda_{0}$ ) is called a microstrip dipole and a broad rectangular antenna is called microstrip patch. Microstrip width has a minor effect on the resonant frequency and radiation pattern of antenna. A larger strip width increases the power radiated and thus decreases resonance resistance and increases bandwidth [8]. We can model a microstrip dipole antenna or a strip dipole antenna that it's dimensions related to thin-wire dipole model as shown in Figure 2.

Microstrip dipole can be designed for the lowest resonant frequency using transmission line model. Thus, $L_{1}=L_{2}=$ $\lambda / 4$ and the gap between each arm of antenna is too small compared to total length of antenna.

Figures 3(a) and 3(b) present a demonstration structure of strip dipole antenna called single-layer strip dipole antenna. Single-layer strip dipole antenna can be added with more layer stack into a structure. This is called double-layers strip dipole antenna, as shown in Figures 3(c) and 3(d). Each layer has the same length when it is straightened but the inner layer will be little longer than the outer layer because the radius of each layer is not the same when it is curved. Accordingly, 


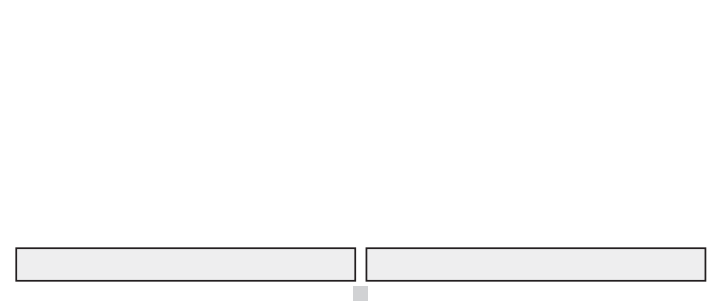

(a) Single-layer straight

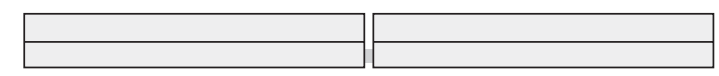

(c) Double-layers straight

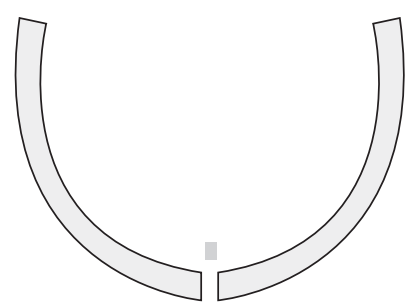

(b) Single-layer curved

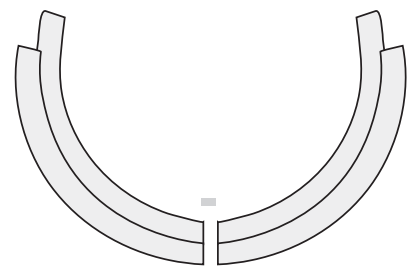

(d) Double-layers curved

FIGURE 3: Schematic of strip dipole antenna.

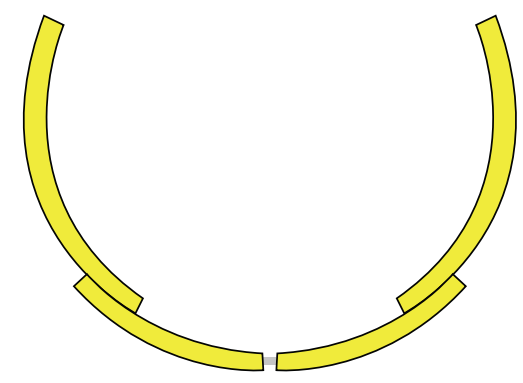

(a) Top view

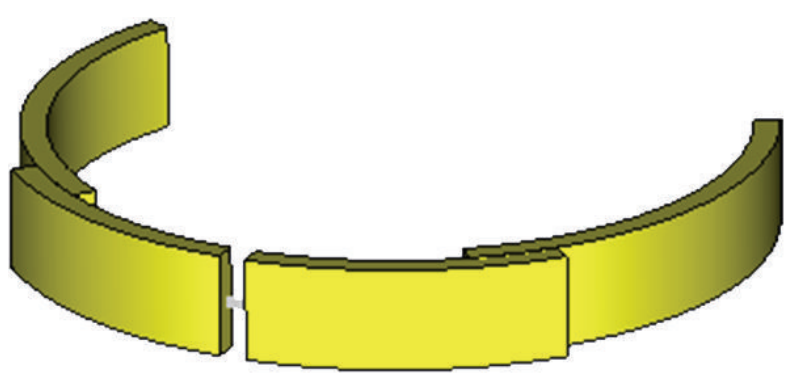

(b) Perspective view

FIGURE 4: Double-layers strip dipole antenna schematic.

we can implement this changeable characteristic for suitable tuning properties of antenna. Normally, when a strip dipole has more length, its resonance frequency will decrease as well [9]. On the other hand, if the length is shorter than the original, the resonance frequency will increase. So, we design a double-layers strip dipole antenna that uses this physical change to compensate curving of antenna. Normally, doublelayers strip dipole antenna has been designed to have shorter outer layer than inner layer as shown in Figure 4 [10].

From Figure 5, the schematic is created to demonstrate how we can calculate effective length when it is curved. The effective length $\left(L_{\text {eff }}\right)$ of slot antenna and double-layers strip dipole antenna when being straightened and curved was reported in $[5,10]$ as

$$
L_{\text {eff }}=2 *\left(L_{11}+\Delta L_{1}+T_{1}+L_{22}\right)
$$

where $L_{m n}$ is the length of strip on layer $m$ section $n$ and $T_{m}$ is thickness of strip on layer $m$. From (2), the effective length of antenna is the same on the left arm and right arm. However,
$\Delta L_{1}$ is an expanded length on layer 1 that came from the effect of difference curving on each layer shown as

$$
\Delta L_{1}=T_{1} \theta_{1}=\frac{T_{1} L_{21}}{R_{2}},
$$

where $L_{21}=R_{2} \theta_{1}$ and $T$ is equal to an antenna thickness on inner layer.

From Figure 5(b), the length of section $L_{11}$ was fixed by physical with supporting structure. So, it would make section $L_{12}$ move away from the center rather than section $L_{22}$. This functioning affects on extension of length $\Delta L_{m}$ occurred on layer $m$. A positive sign of $\Delta L_{m}$ in (2) mean to the normal length has been extended. Figure 5 shows that sections $L_{11}$ and $L_{22}$ must have some extended length $\left(X_{1}\right)$ to cover a difference length that occurred from curved effect for making both layers contacted to each other.

Next, we adapted a flexible copper-clad laminate as raw material because of flexibility and it is easy to be fabricated. There are many types of materials that are normally used for RFID antenna. For our design, a Pyralux AP9121R of 


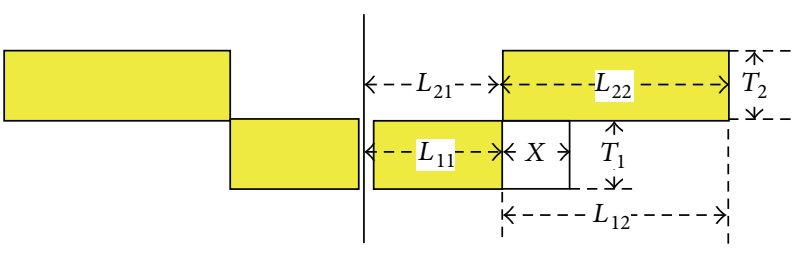

(a) Straight

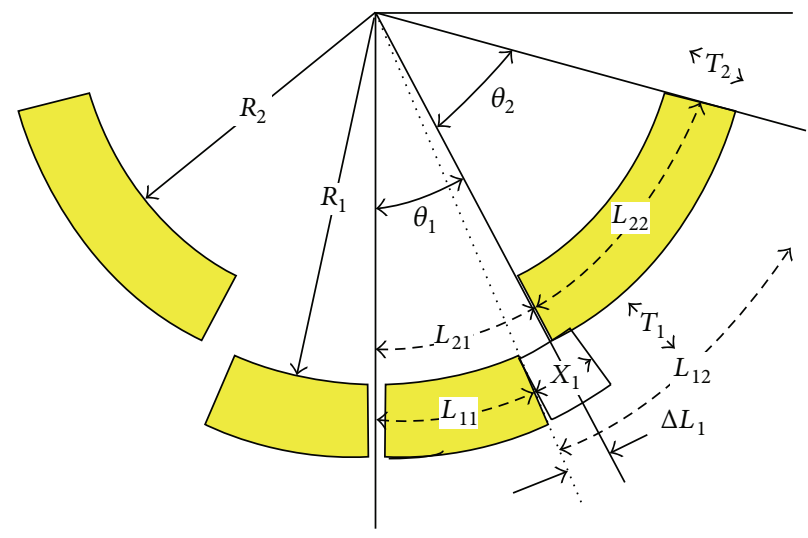

(b) Curved

FIGURE 5: Schematic of double-layers strip dipole.

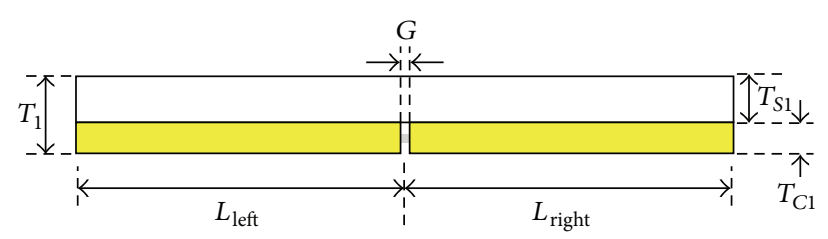

(a) Straight

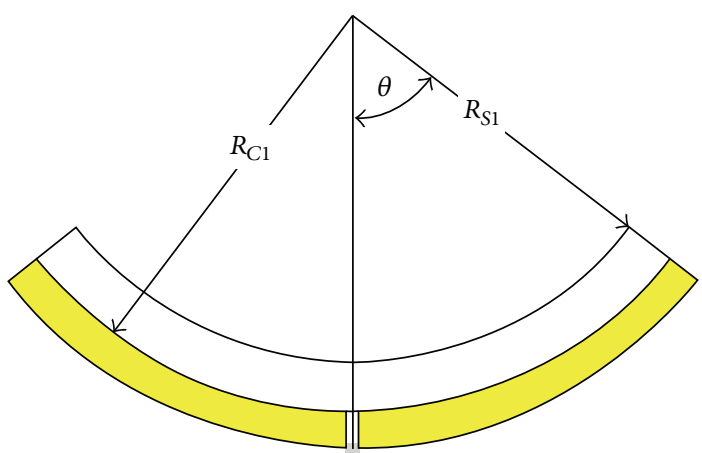

(b) Curved

FIGURE 6: Schematic of single-layer strip dipole antenna design with flexible copper-clad laminate.

TABLE 1: The dimension parameters of multilayer strip dipole antenna (unit: millimeter(s)).

\begin{tabular}{|c|c|c|c|c|c|}
\hline \multirow{2}{*}{ Parameter name } & \multicolumn{5}{|c|}{ Antenna type } \\
\hline & Single-layer & Double-layers & Triple-layers & Four-layers & Five-layers \\
\hline$W$ & 10.00 & 10.00 & 10.00 & 10.00 & 10.00 \\
\hline$T_{C 1-C 5}$ & 0.035 & 0.035 & 0.035 & 0.035 & 0.035 \\
\hline$T_{S 1-S 5}$ & 0.051 & 0.051 & 0.051 & 0.051 & 0.051 \\
\hline G & 1.00 & 1.00 & 1.00 & 1.00 & 1.00 \\
\hline $\mathrm{O}_{2-5}$ & 0.00 & 5.00 & 5.00 & 5.00 & 5.00 \\
\hline$L_{11}$ & $72.625^{*}$ & 15.00 & 15.00 & 15.00 & 15.00 \\
\hline$L_{21}$ & - & 15.00 & 15.00 & 15.00 & 15.00 \\
\hline$L_{22}$ & - & $51.87^{*}$ & 10.00 & 10.00 & 10.00 \\
\hline$L_{31}$ & - & & 25.00 & 25.00 & 25.00 \\
\hline$L_{32}$ & - & & $42.62^{*}$ & 10.00 & 10.00 \\
\hline$L_{41}$ & - & & & 35.00 & 35.00 \\
\hline$L_{42}$ & - & & & $33.30^{*}$ & 10.00 \\
\hline$L_{51}$ & - & & & & 45.00 \\
\hline$L_{52}$ & - & & & & $23.27^{*}$ \\
\hline
\end{tabular}

\footnotetext{
*optimized value from simulation at $920 \mathrm{MHz}$.
} 


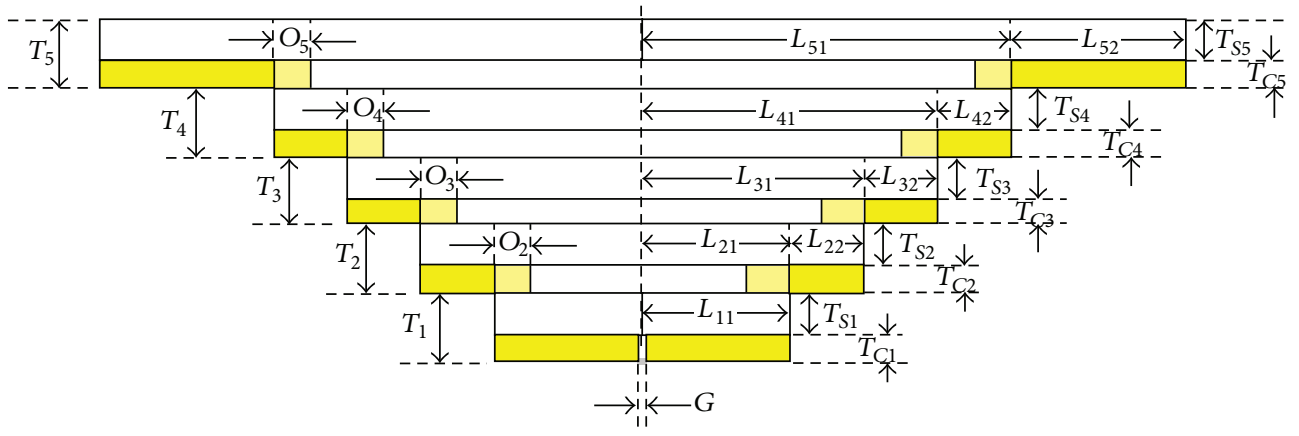

(a) Five-layers straight

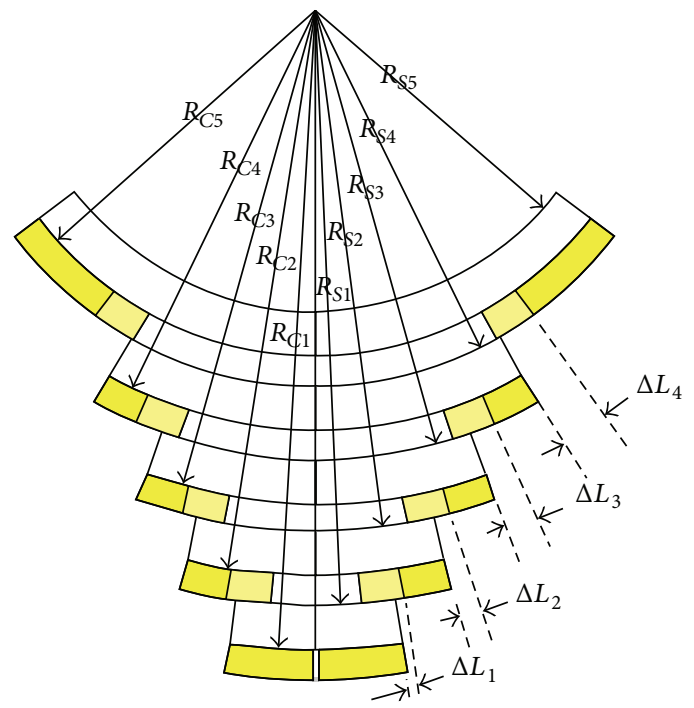

(b) Five-layers curved

FIGURE 7: Five-layers strip dipole antenna schematic.

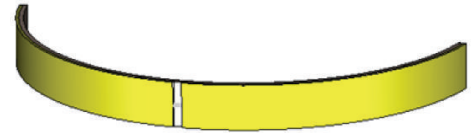

(a) Single-layer strip dipole antenna

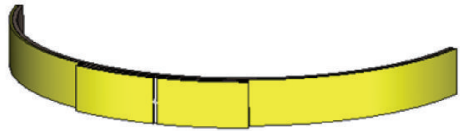

(b) Double-layers strip dipole antenna

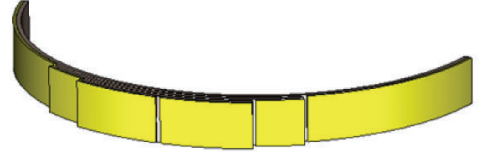

(c) Triple-layers strip dipole antenna

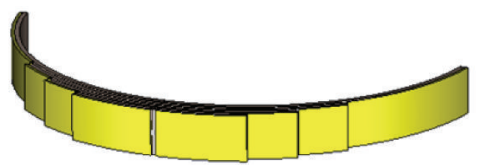

(d) Four-layers strip dipole antenna

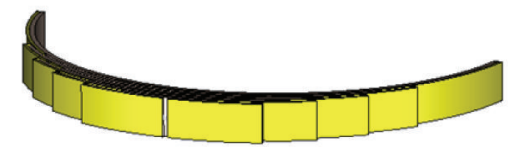

(e) Five-layers strip dipole antenna

FIGURE 8: Multilayers strip dipole antenna model (not true scale).

DuPont is selected [11]. A copper-clad laminate is an allpolyimide composite of polyimide film bonded to copper foil. This material system is ideal for multilayer flex and rigid flex applications which require advanced material performance, temperature resistance, and high reliability. It has dielectric constant, dielectric thickness, and copper clad thickness equal to 3.4, 0.051 millimeters and 0.035 millimeters, respectively.
However, it seems that flexible copper-clad laminate has a very small thickness to make a lot of extend length comparing to double-layers strip dipole made from pure copper sheet when it is curved. So, it is possible that we can stack it to make a multilayer strip dipole antenna with this material to increase thickness and make a better frequency response of antenna compared to an original design with a double-layers strip dipole antenna. 


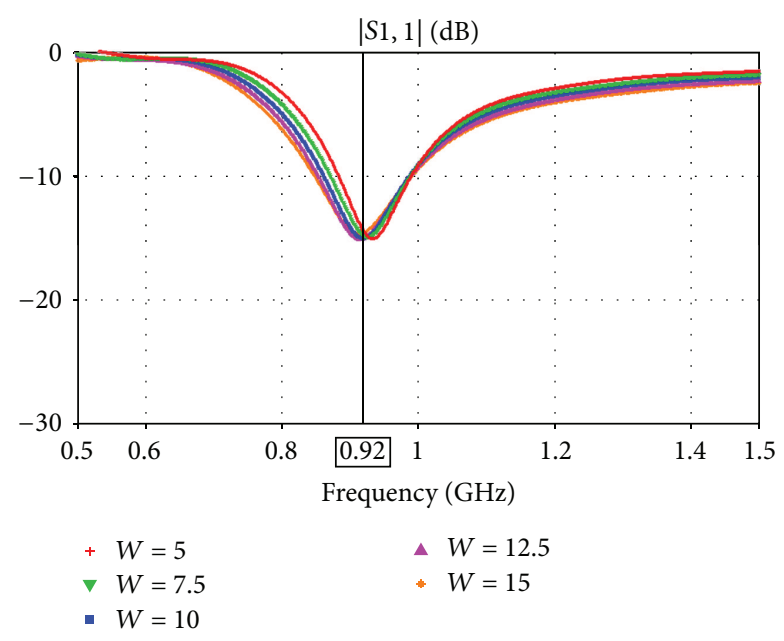

FIGURE 9: Resonance frequency of single-layer strip dipole antenna with difference width (millimeters).

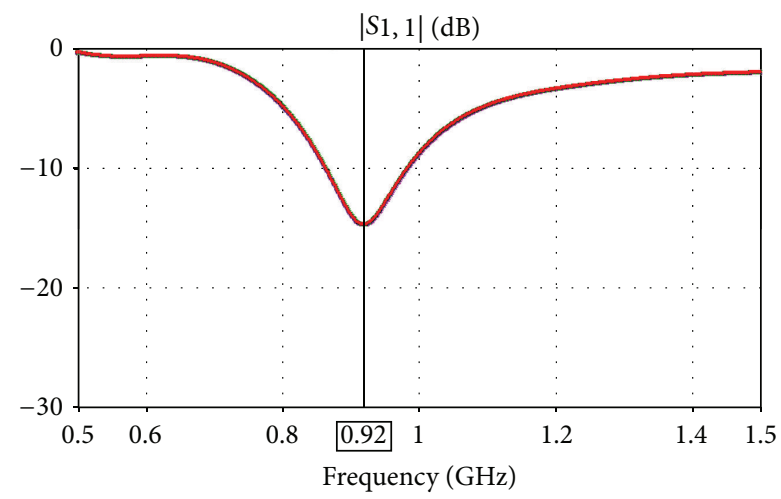

$$
\begin{array}{ll}
+O=3 & \text { A }=6 \\
\nabla O=4 & * O=7
\end{array}
$$$$
\text { - } \mathrm{O}=5
$$

FIGURE 10: Resonance frequency of five-layers strip dipole antenna with difference overlap length (millimeters).

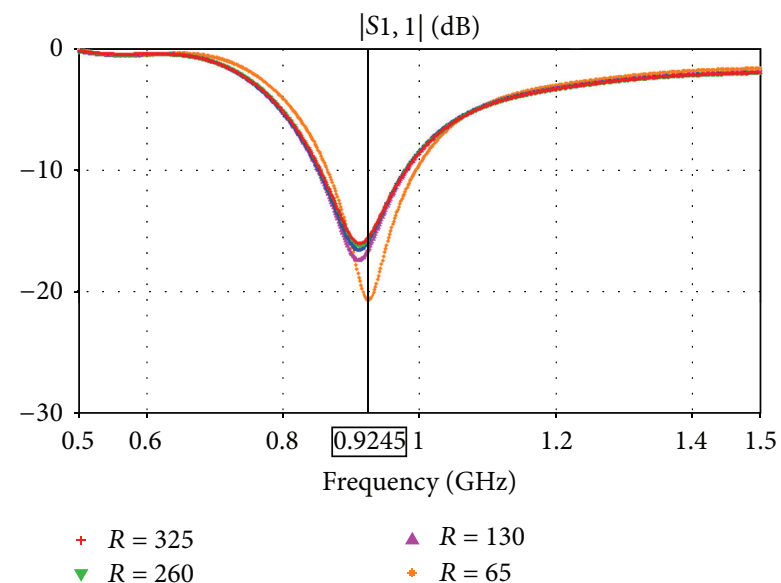

FIGURE 11: Resonance frequency of single-layer strip dipole antenna with difference curving radius (millimeters).

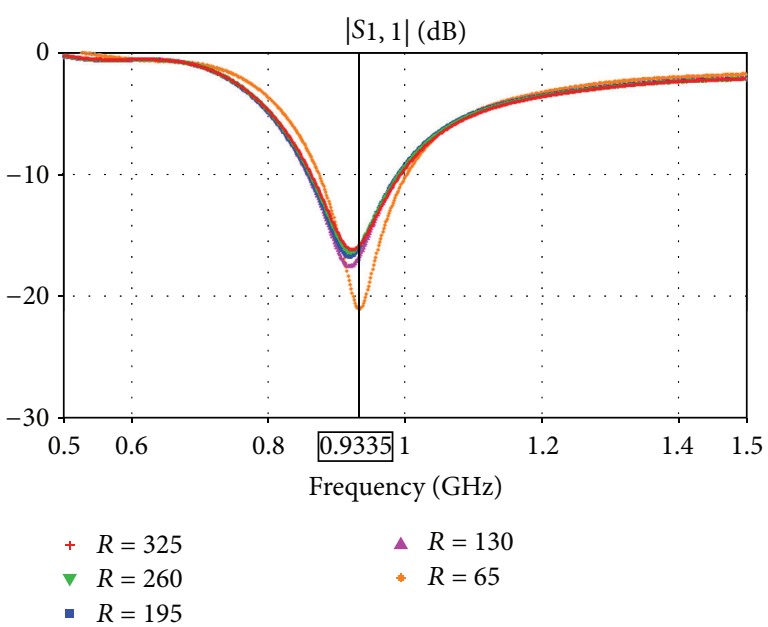

FIGURE 12: Resonance frequency of double-layers strip dipole antenna with difference curving radius (millimeters).

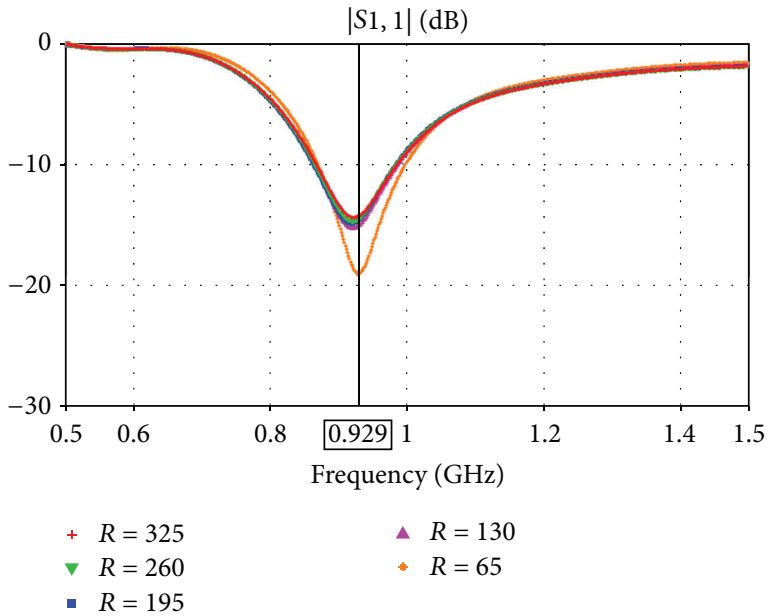

FIGURE 13: Resonance frequency of triple-layers strip dipole antenna with difference curving radius (millimeters).

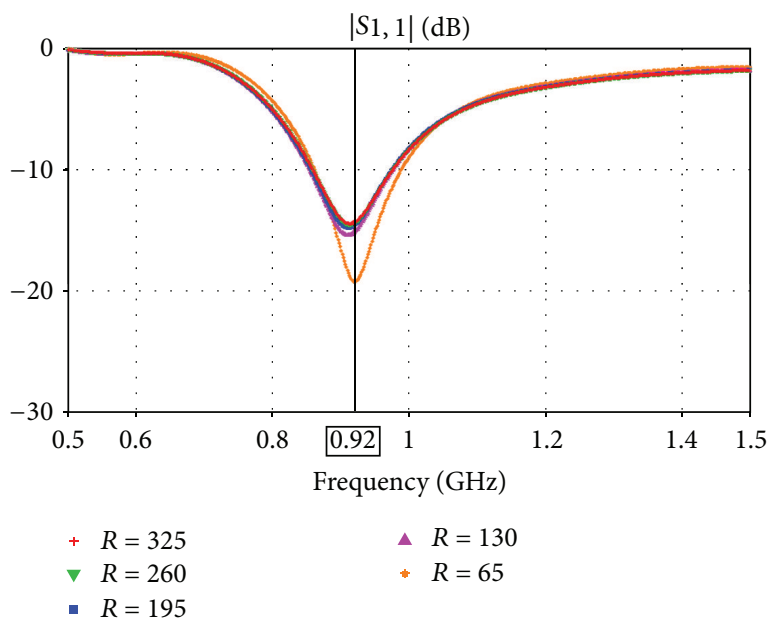

FIGURE 14: Resonance frequency of four-layers strip dipole antenna with difference curving radius (millimeters). 


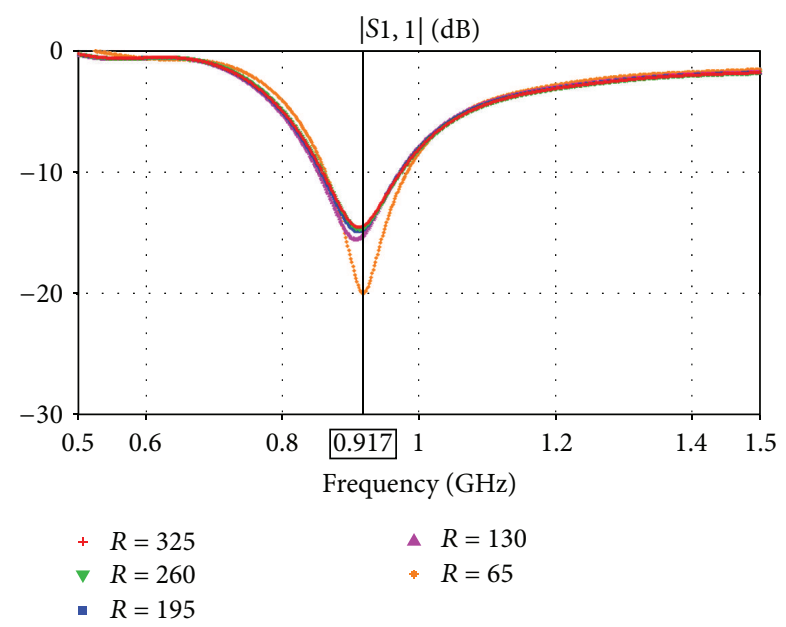

FIGURE 15: Resonance frequency of five-layers strip dipole antenna with difference curving radius (millimeters).
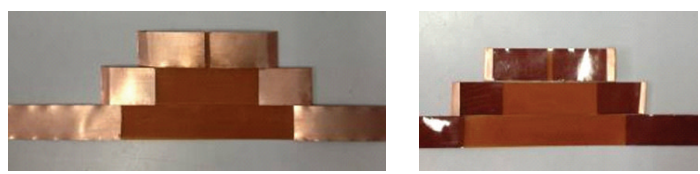

(a) Triple-layers strip dipole antenna
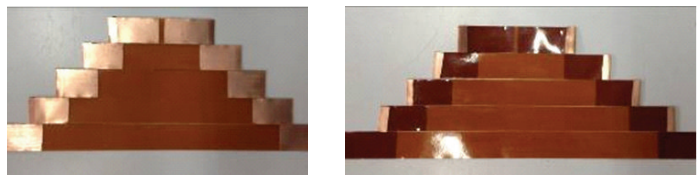

(b) Five-layers strip dipole antenna

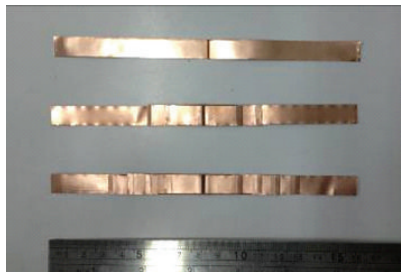

(c) Prototype of multilayer strip dipole antennas after assembly with glue

FIGURE 16: Prototype of multilayer strip dipole antennas.

TABLE 2: Curving ratio and actual curving radius.

\begin{tabular}{lc}
\hline Curving ratio & Actual curving radius $(\mathrm{mm})$ \\
\hline $0.2 \lambda_{0}$ & 65 \\
$0.4 \lambda_{0}$ & 130 \\
$0.6 \lambda_{0}$ & 195 \\
$0.8 \lambda_{0}$ & 260 \\
$1.0 \lambda_{0}$ & 325 \\
\hline
\end{tabular}

So, when it has a lot of layers, it makes a bit longer process to make an antenna. This means to a limitation of multilayer with stacking technique which will be decided for how many layers for fabrication to be capable to perform with good economic value.

Figure 6 shows a model for single-layer of flexible copperclad laminate strip antenna. We can call them in general term as a multilayer strip dipole if we stack a flexible copper-clad laminate more than two layers. A demonstrated diagram to calculate effective length of five-layers strip dipole antennas is shown in Figure 7.

From Figure 7 the schematic of five-layers strip dipole antenna is demonstrated, with the effective length written as

$$
\begin{array}{r}
L_{\text {eff }}=2 *\left\{L_{11}+\Delta L_{1}+T_{1}+L_{22}+\Delta L_{2}+T_{2}+L_{32}\right. \\
\left.+\Delta L_{3}+T_{3}+L_{42}+\Delta L_{4}+T_{4}+L_{52}\right\},
\end{array}
$$

where

$$
\begin{array}{rlrl}
\Delta L_{1} & =\frac{T_{1} L_{21}}{R_{C 2}}, & \Delta L_{2}=\frac{T_{2} L_{31}}{R_{C 3}}, \\
\Delta L_{3}=\frac{T_{3} L_{41}}{R_{C 4}}, & \Delta L_{4}=\frac{T_{4} L_{51}}{R_{C 5}} .
\end{array}
$$

The thickness of each layer $\left(T_{M}\right)$ can be calculated by total thickness of flexible copper-clad laminate that equals thickness of copper layer $\left(T_{C M}\right)$ plus thickness of dielectric substrate $\left(T_{S M}\right)$ where $M$ is number of layers. Otherwise, a radius of curved on each layer can be used as a radius of copper layer $\left(R_{C M}\right)$. So, we can find out an extended length of each layer for five-layers strip dipole antenna by (5).

\section{Simulation Results}

Figure 8 shows a schematic of the multilayer strip dipole antenna simulated by using CST-Microwave Studio high frequency simulator [12]. The antenna is laid on $X Y$-plane and curve in $X Z$-plane. Some of the antenna parameters affected the resonance frequency of the antenna. The comparable result found that only one parameter changed when the other was fixed. By the way, the strip width will mostly affect the frequency bandwidth [9].

Typically, strip width is less than $0.05 \lambda_{0}$ for a strip dipole [9] that is around 16.3 millimeters for center frequency at $920 \mathrm{MHz}$. Figure 9 shows effects of strip width that extend antenna bandwidth. So, a strip width is set to 10 millimeters for a proper size for use and has enough bandwidth covering UHF-RFID standard ( 850 to $960 \mathrm{MHz}$ ). The bandwidth of UHF-RFID standard in Thailand is $920-925 \mathrm{MHz}$ [13].

Otherwise, this design will not be affected by the overlap length. If they are changed, the overlap lengths $\left(O_{M}\right)$ are varying from $3,4,5,6$, and 7 millimeters, respectively. The resonance frequency is still the same at $920 \mathrm{MHz}$ as shown in the simulation result in Figure 10.

Flexible copper-clad laminate has copper layer of 0.035 millimeters in thickness; the substrate thickness is 0.051 millimeters. The total length of the antenna will be used for a quarter wavelength, which is equal to $81.25 \mathrm{~mm}$. but the final length will be changed depending on simulation result to provide frequency response at $920 \mathrm{Mhz}$. The other lengths of all section are shown in Table 1, where antenna type is a 


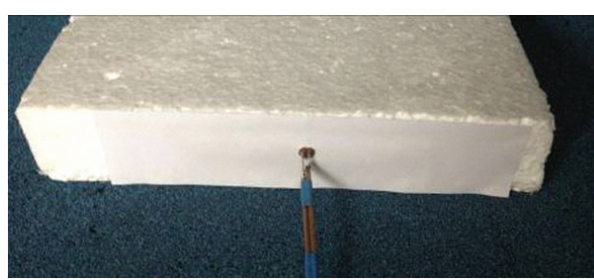

(a) Straight

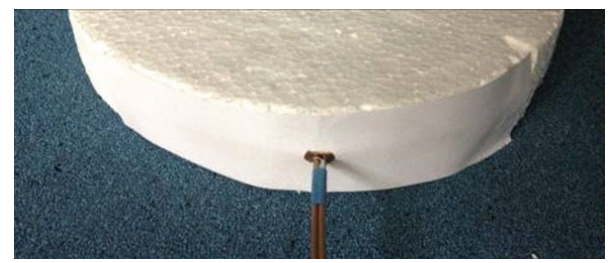

(b) Curved at $R=130 \mathrm{~mm}$

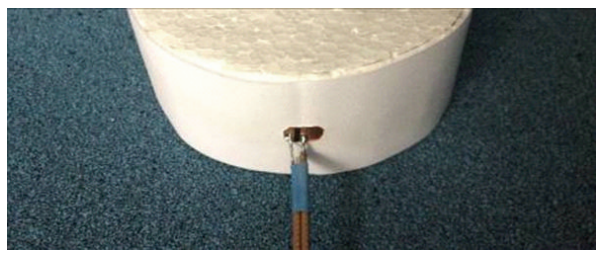

(c) Curved at $R=65 \mathrm{~mm}$

FIGURE 17: Multilayer strip dipole antenna with balun and cover paper used for measurement.

TABLE 3: Resonance frequency of strip dipole antenna with difference curving radius.

\begin{tabular}{lccccc}
\hline \multirow{2}{*}{ Curving radius $(\mathrm{mm})$} & \multicolumn{3}{c}{ Resonance frequency $(\mathrm{MHz})$} \\
& Single-layer & Double-layers & Triple-layers & Four-layers & Five-layers \\
\hline 325 & 912.50 & 924.50 & 923.00 & 914.00 & 911.00 \\
260 & 912.50 & 921.50 & 920.00 & 912.50 & 912.50 \\
195 & 911.00 & 920.00 & 918.50 & 911.00 & 911.00 \\
130 & 911.00 & 920.00 & 920.00 & 911.00 & 906.50 \\
65 & 924.50 & 933.50 & 929.00 & $\mathbf{9 1 4 . 7 5}$ & $\mathbf{9 1 3 . 0 0}$ \\
Average & $\mathbf{9 1 5 . 2 5}$ & $\mathbf{9 2 3 . 2 5}$ & $\mathbf{9 2 1 . 7 5}$ & $\mathbf{4 . 2 2}$ & $\mathbf{4 . 8 1}$ \\
SD & $\mathbf{5 . 6 5}$ & $\mathbf{5 . 3 2}$ & $\mathbf{3 . 8 4}$ & & $\mathbf{4 5 0}$ \\
\hline
\end{tabular}

single-layer, double-layers, triple-layers, four-layers, and fivelayers strip dipole antenna.

The simulation result of using different curved radius is equal to a curving ratio multiplied by wavelength $\left(\lambda_{0}\right)$ of center frequency. In this case, center frequency is $920 \mathrm{MHz}$. So, a wavelength is equal to 325 millimeters that converts to actually curving radius as shown in Table 2 .

The radius of curve has been changed in order to observe the reflection coefficient (S11) and resonance frequency. The result of the simulation with different curving ratios of singlelayer, double-layers triple-layers, four-layers, and five-layers strip dipole antenna are shown in Figures 11, 12, 13, 14, and 15, respectively.

For practical, a multilayer antenna must have a structural supported sheet cover on front of antenna to make each layer contacted together. We have used a thin paper for this purpose. Property of paper has a dielectric constant of 2.3 and thickness around $0.038 \mathrm{~mm}$. So, it makes a small frequency shift when we compare to an original antenna without paper cover. Finally, we add a $0.038 \mathrm{~mm}$ thickness paper including in our model to simulation that has size $40 \times 16 \mathrm{~mm}$.

Basically, resonance frequency increases higher when a single-layer strip dipole antenna is curved as reported in $[10,14]$. So, a simulation results of all strip dipole antenna are shown in Table 3.
According to these simulation results, it could be summarized that the resonance frequency of antenna and curving ratio is more stable when the layers match as shown in Table 3. For example, on single-layer strip dipole antenna, the resonance frequency has been changed from $920.0 \mathrm{MHz}$ to $915.25 \mathrm{MHz}$ for average when curving with different curved ratios. Its resonance frequency has been shifted to $4.75 \mathrm{MHz}$ or 4.75 percent of bandwidth (UHF-RFID standard $850 \mathrm{MHz}-960 \mathrm{MHz})$.

Then, the average of the resonance frequency changed from $920 \mathrm{MHz}$ for double-layers, triple-layers, four-layers and five-layers strip dipole antenna was $3.25 \mathrm{MHz}, 1.75 \mathrm{MHz}$, 4.75 MHz and $3.00 \mathrm{MHz}$, respectively. It shows that a standard deviation of the resonance frequency of single-layer, double-layers, triple-layers, four-layers, and five-layers strip dipole antenna was 5.65, 5.32, 3.84, 4.22, and 4.81, respectively. So, the most minimum change of resonance frequency with difference curving radius is triple-layers strip dipole antenna.

\section{Measurement Results}

From simulation result, we choose to represent only three types of them to make a prototype that was single-layer, 
triple-layers, and five-layers. The prototype antennas were fabricated from flexible copper-clad laminate that has dimension and electrical properties is the same as the simulation model. The prototype antenna was made from flexible copper-clad laminate as shown in Figure 17. A network analyzer HP8722D used for measurement S11 results in anechoic chamber.

However, the edge of each layer will be flipped over and bent backward for joining to another layer to make a physical contact on entire antenna. Next, each of the layers was fixed together by glue at the center. Finally, we have a prototype antennas as shown in Figure 16.

In order to be able to measure the dipole antenna via coaxial cable, a balance-to-unbalance transformer balun is used. A quarter-wave coaxial balun or folded balun is relatively easily constructed [15]. A folded balun can be constructed using extra piece of coaxial cable. It is connected between feeding coaxial cable and the antenna side to the inner conductor of the feed. This extra cable should be a quarter of wave length long. Therefore, this type of balun has no effect on input impedance. Further more, the quarter wavelength line induces another current on the outside of the outer conductor, which canceled the unbalanced current. However, we connected this balun with a SMA connector for measurement as shown in Figure 17. It is not part of the strip dipole antenna, and it can be considered an external module used just for measurement purposes.

From measurement results, a resonance frequency of single-layer strip dipole antenna is at $920.0 \mathrm{MHz}$. The resonance frequency has been shifted to $912.5 \mathrm{MHz}$ with curving radius of 130 millimeters and $924.9 \mathrm{MHz}$ with curving radius 65 millimeters. Then, a triple-layers strip dipole antenna has resonance frequency at $920.0 \mathrm{MHz}$ when being straightened. The resonance frequency has been shifted to $920.9 \mathrm{MHz}$ and $918.5 \mathrm{MHz}$ when it curved at 130 millimeters and 65 millimeters, respectively.

Finally, a five-layers strip dipole antenna has resonance frequency of $920.0 \mathrm{MHz}$ when it is straightened. The resonance frequency has been shifted to $905.4 \mathrm{MHz}$ and $917.1 \mathrm{MHz}$ when it curved at 130 millimeters and 65 millimeters, respectively. This result agrees with simulation result in Section 3 that triple-layers and five-layers strip dipole has improved the change of resonance frequency due to the antenna to be curved.

\section{Conclusion}

This paper presents a novel multilayer strip dipole antenna using stacking technique. The proposed antenna has uses flexible copper-clad laminate, which this flexibility is the advantages to be applied for the application of RFID tags. The simulation of improvement is achieved when the multilayer strip dipole antenna is curved. A design of antenna and effects of size which varied by frequency of antenna have been presented. The results of simulation will be implemented to design an appropriate and efficient antenna. According to the simulation, the multilayer strip dipole antenna is more attractive than single-layer strip dipole antenna because it performs higher stability of resonance frequency while curving. In addition, the deviation of resonance frequency for multilayer strip dipole antenna when it curved is less than singlelayer strip dipole antenna with various curving ratios. This designed technique could be confirmed by a measurement result from a prototype antenna that agrees with simulation results. Hence, the antenna must be designed to match with specific characteristics of object surfaces including material properties in order to improve RFID system performance.

\section{Conflict of Interests}

The authors declare that there is no conflict of interests regarding the publication of this paper.

\section{Acknowledgments}

This work was supported by Suranaree University of Technology (SUT) and by the Office of the Higher Education under NRU Project of Thailand. The authors deeply appreciate the valuable comments of the reviewers and recommends to be advantageous for revisions this paper.

\section{References}

[1] K. Finkenzeller, RFID Handbook: Fundamentals and Applications in Contactless Smart Cards, Radio Frequency Identification and Near-Field Communication, John Wiley \& Sons, New York, NY, USA, 2010.

[2] Z. N. Chen, Antennas for Portable Devices, John Wiley \& Sons, New York, NY, USA, 2007.

[3] D. M. Dobkin, The RF in RFID: Passive UHF RFID in Practice, Newnes, Newton, Mass, USA, 2007.

[4] P. Salonen and Y. Rahmat-Samii, "Textile antennas: effects of antenna bending on input matching and impedance bandwidth," IEEE Aerospace and Electronic Systems Magazine, vol. 22, no. 3, pp. 10-14, 2007.

[5] A. Galehdar and D. V. Thiel, "Flexible, light-weight antenna at $2.4 \mathrm{GHz}$ for athlete clothing," in Proceedings of the IEEE Antennas and Propagation Society International Symposium, pp. 4160-4163, June 2007.

[6] J. D. Kraus, "Heinrich Hertz-theorist and experimenter," IEEE Transactions on Microwave Theory and Techniques, vol. 36, no. 5, pp. 824-829, 1988.

[7] C. Butler, "The equivalent radius of a narrow conducting strip," IEEE Transactions on Antennas and Propagation, vol. 30, no. 4, pp. 755-758, 1982.

[8] M. H. Jamaluddin, M. K. A. Rahim, M. Z. A. A. Aziz, and A. Asrokin, "Microstrip dipole antenna analysis with different width and length at $2.4 \mathrm{GHz}$," in Proceedings of the Asia-Pacific Conference on Applied Electromagnetics (APACE '05), pp. 41-44, December 2005.

[9] R. Garg, P. Bhartia, I. Bahl, and A. Ittipiboon, Microstrip Antenna Design Handbook, Artech House, Norwood, Mass, USA, 2001.

[10] C. Saetiaw, C. Thongsopa, and A. Intarapanich, "RFID tag antenna design using flexible double-layer strip dipole antenna," in Proceedings of the International Symposium on Antennas and Propagation, Jeju, Republic of Korea, 2011. 
[11] DuPont, "flexible circuit materials: Technical Data Sheet," http://www.dupont.com/.

[12] CST-Microwave Studio, 2009.

[13] "Regulatory status for using RFID in the EPC Gen 2 band (860 to $960 \mathrm{MHz}$ ) of the UHF spectrum," http://www.gs1.org/.

[14] J. Siden, P. Jonsson, T. Olsson, and G. Wang, "Performance degradation of RFID system due to the distortion in RFID tag antenna," in Proceedings of the 11th International Conference on Microwave and Telecommunication Technology, pp. 371-373, 2001.

[15] C. A. Balanis, Antenna Theory: Analysis and Design, John Wiley \& Sons, Hoboken, NJ, USA, 2005. 

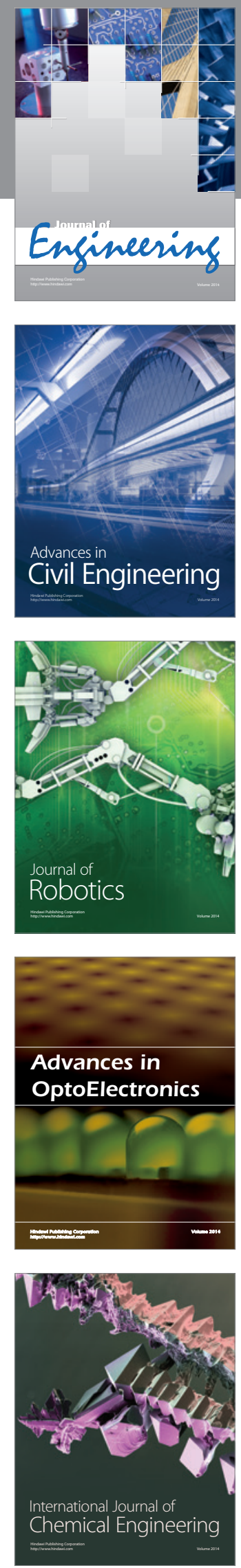

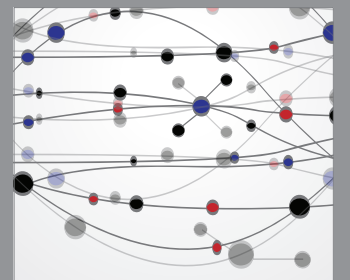

The Scientific World Journal
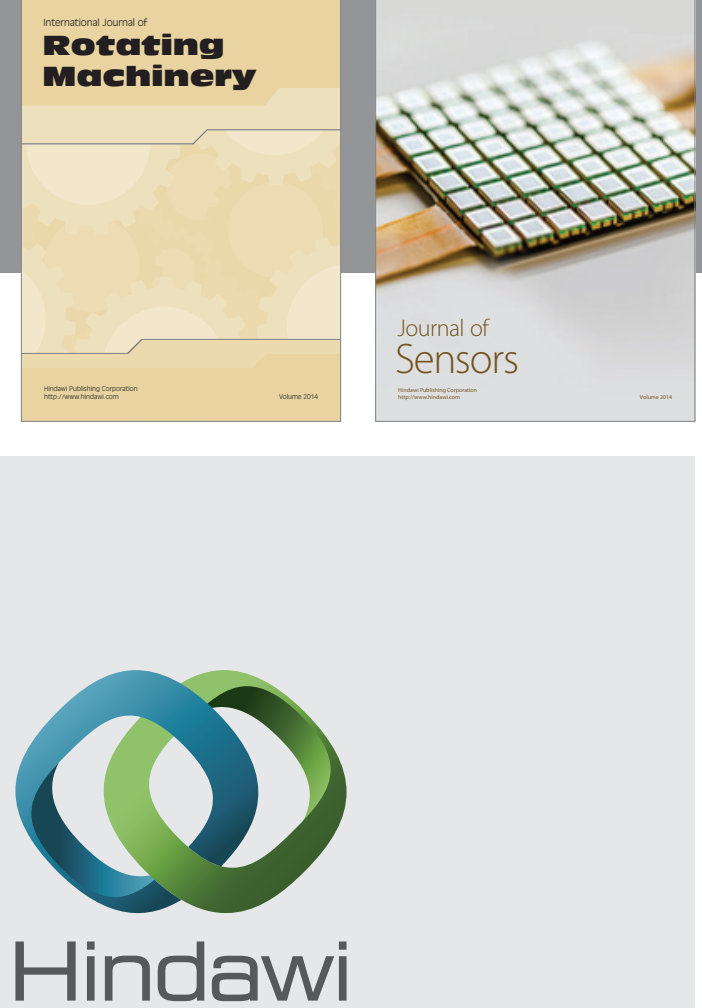

Submit your manuscripts at http://www.hindawi.com
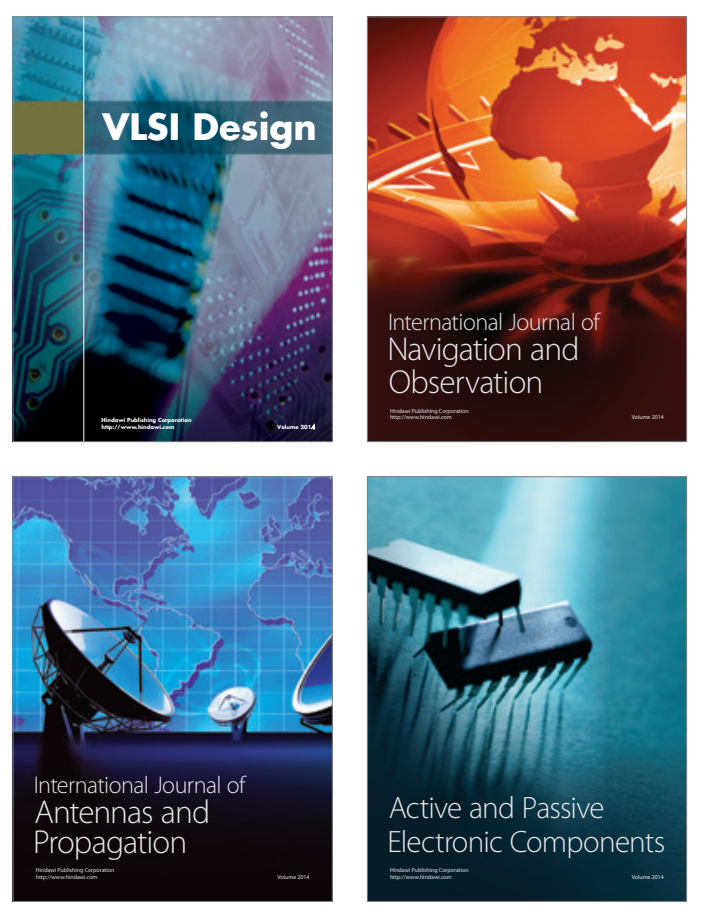
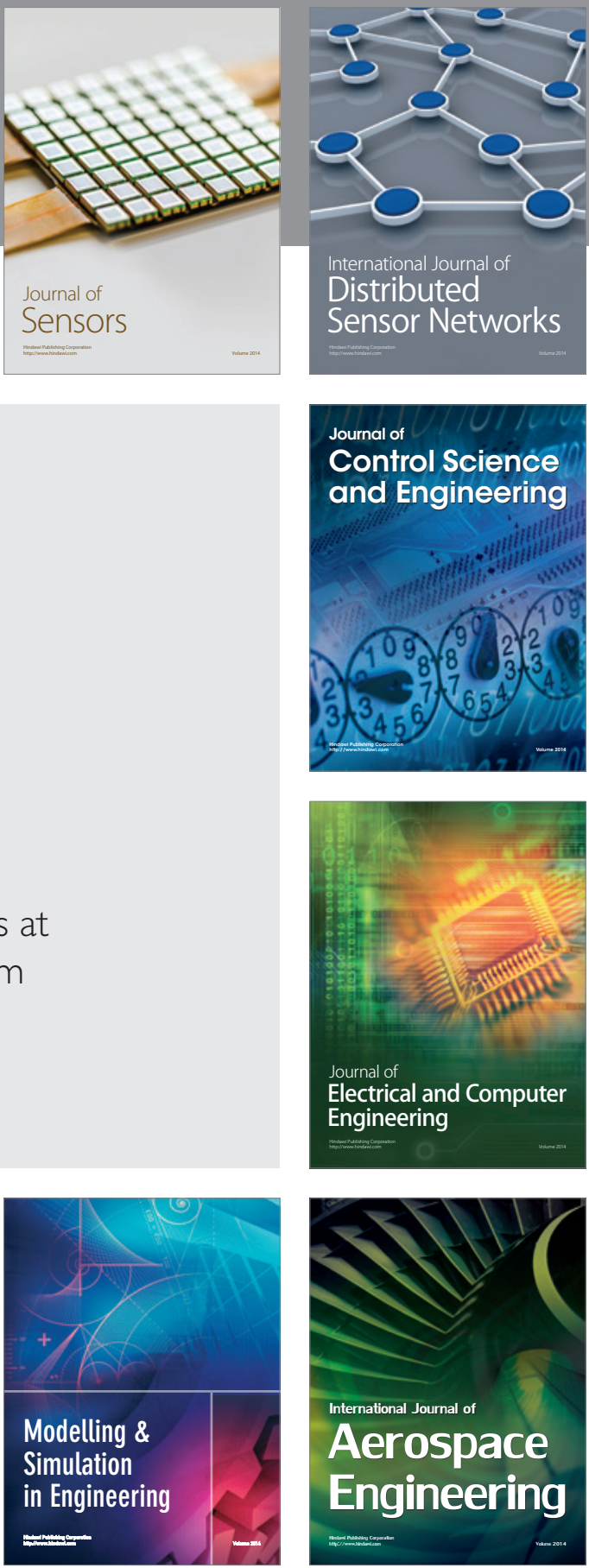

Journal of

Control Science

and Engineering
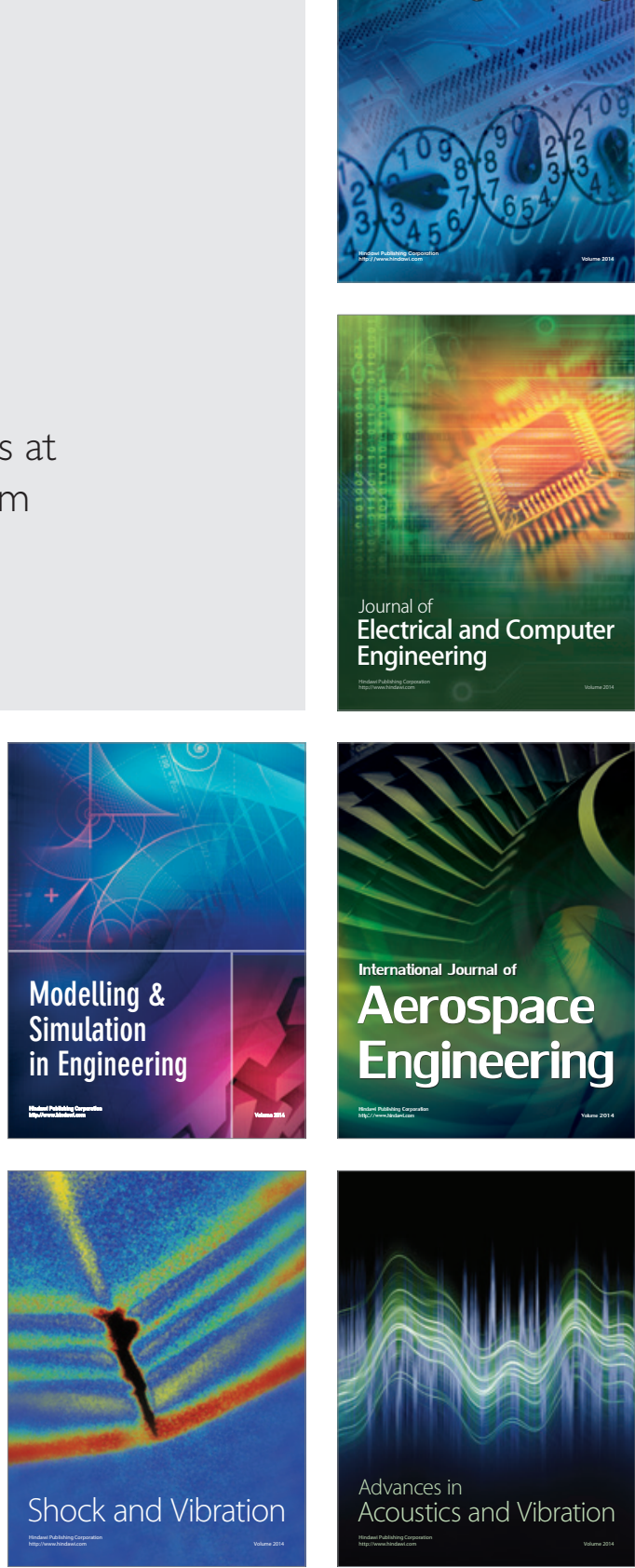